\title{
Fenologia dos Braconidae (Hymenoptera, Ichneumonoidea) da Área de Proteção Ambiental (APA) de Descalvado, SP
}

\author{
Kátia Resende Netto Cirelli ${ }^{1}$ \\ Angélica Maria Penteado-Dias ${ }^{2}$
}

\begin{abstract}
Phenology of the Braconidae (Hymenoptera, Ichneumonoidea) at Área de Proteção Ambiental of Descalvado, São Paulo State. The flight phenology of the Braconidae fauna was conducted using Malaise traps in five sites of the Environmental Protected Area of Descalvado. A total of 2,792 specimens, representing 22 subfamilies, was sampled throughout a period of sixteen months from May, 1999 to August, 2000. The traps catches for koinobionts peak was in August, 1999 a month before of the idiobionts peak and in June, 2000 both peaks were simultaneous. The phenological peaks of dominant koinobiont taxa (Microgastrinae) were similar to all koinobionts, and the peaks of dominant idiobiont taxa (Doryctinae) were similar to all idiobionts, as well. December, 1999 and February, 2000 were the richer months in number of subfamilies ( $\mathrm{N}=19$ and 18, respectivaly), corresponding to the same period when the subfamilies which potentially attack Lepidoptera where collected. The number of females was superior to the number of males and this relation was more accentuated in koinobionts. The peaks of both females and males were similar to dominate koinobiont and idiobionte taxa.
\end{abstract}

KeYwOrds. Braconidae; Brazil; flight fenology, fragmentary natural environments, parasitoid.

\section{INTRODUÇÃO}

Os Braconidae são parasitóides que constituem, depois dos Ichneumonidae, a família mais numerosa, com cerca de 40.000 espécies (ACHTERBERG 1988). São insetos pequenos e ativos que, como os demais parasitóides, apresentam estágios larvais que se desenvolvem sobre ou no interior de outros artrópodos, geralmente insetos. Apresentam grau variável de especificidade de hospedeiro sendo que a maioria de suas espécies ataca insetos fitófagos principalmente Lepidoptera, Diptera ou Coleoptera. Algumas espécies atacam ovos, pupas e até mesmo adultos de seus hospedeiros. O hiperparasitismo é extremamente raro. As formas adultas são de vida livre e alimentam-se de fluidos vegetais (mel e pólen), nutrientes absorvidos, principalmente na fase imatura (JERVIS et al. 1993), ou ingerem hemolinfa quando da oviposição. Entretanto, o desenvolvimento larval resulta na morte do hospedeiro (GODFRAY 1994).

Entre os Braconidae, os idiobiontes não permitem que seus hospedeiros se desenvolvam depois de parasitados; os coinobiontes, por sua vez, o fazem até que eles atinjam tamanho e estágios predeterminados (AsKew \& SHAw 1986). Os idiobiontes utilizam como hospedeiros larvas de últimos instares, pré-pupas ou pupas principalmente de Lepidoptera, Diptera ou Coleoptera que geralmente estão em locais ocultos. A fêmea idiobionte precisa localizar e ter acesso a eles e, para isso, utiliza o ovipositor que geralmente é adaptado para a perfuração do substrato no qual o hospedeiro está abrigado. As fêmeas usualmente injetam veneno que matam ou, às vezes, os paralisam antes da oviposição propriamente dita. As larvas idiobiontes podem se desenvolver sobre diversos tipos de hospedeiros encontrados em determinado substrato, sendo portanto, consideradas generalistas e, a grande maioria, ectoparasitóides (Shaw \& HUdDLESTON 1991). Os coinobiontes ovipositam no interior de (raramente sobre) larvas de primeiros instares, ou ovos de seus hospedeiros (principalmente de Lepidoptera, Diptera ou Coleoptera) que geralmente estão expostos. As fêmeas coinobiontes não possuem ovipositor adaptado à perfuração de substratos. As larvas coinobiontes geralmente são pequenas e se desenvolvem devagar, poupando o hospedeiro até o estágio de pupa, quando então consomem suas partes vitais (ТотнILl apud GAUld 1987); são

1. Produzir - Gestão Agropecuária e ambiental LTDA. Av. Bom Jesus, 711, 13690-000 Descalvado-SP, Brasil.

Endereço eletrônico: katiacirelli@aol.com

2. Universidade Federal de São Carlos, Departamento de Ecologia e Biologia Evolutiva. Caixa Postal 676, 13565-905 São Carlos - SP, Brasil.

Endereço eletrônico: angelica@ power.ufscar.br 
fisiologicamente mais adaptadas ao seu hospedeiro (SALT 1968; DOVER \& VINSON 1990) e consideradas especialistas (SHAW \& HudDleston 1991). O sucesso do parasitismo está relacionado com a larva do parasitóide, isto é, sua adequação ao hospedeiro e sua regulação com o mesmo (VINSON, 1981 apud ALPHEN \& VET, 1986).

A maioria das espécies de Braconidae é cosmopolita, de hábito diurno (GAUld 1991; Lewis \& WhitFiEld 1999), de tamanho pequeno a moderado e capazes de vôos direcionados. Estudos conduzidos por Juillet $(1960,1964)$ na América do Norte, demonstraram que os braconídeos tendem a ser mais ativos onde a vegetação é parcialmente aberta, em temperatura alta, baixa umidade e ventos com baixa velocidade, condições abióticas ótimas que os associam aos insetos-praga de agroecossistemas. O ciclo de vida é variável, pois estes insetos atacam hospedeiros em determinados estágios de sua vida, geralmente no estágio larval, quando ele é abundante. JERvis et al. (1993) observaram que também ocorre um sincronismo espaço-temporal do período de vôo dos braconídeos (e demais grupos de parasitóides adultos) com o período de floração da vegetação, que são fontes energéticas e protéicas para a sua sobrevivência, ovogênese e conseqüente sucesso na busca e obtenção de hospedeiros.

Muito tem se discutido sobre os fatores biológicos e/ou climáticos que governam a abundância dos parasitóides, incluindo os braconídeos (Nealis 1988; Gauld 1991; Thangavelu 1993). Entretanto, pesquisas sobre parasitóides são limitadas pelo seu comportamento complexo, pequeno tamanho e por carência de coleções entomológicas, resultando no ainda escasso conhecimento do grupo(GAULD \& Bolton 1988), tanto biológico como taxonômico.

O objetivo deste trabalho é estudar a fenologia (distribuição temporal) (WoLdA 1988) das subfamílias de Braconidae amostradas na Área de Proteção Ambiental (APA) de Descalvado por Malaise durante o período de maio de 1999 a agosto de 2000, e identificar, com base na literatura, os seus

Tabela I. Abundância das subfamílias de Braconidae capturadas na APA de Descalvado, SP através de armadilhas Malaise, durante o período de maio de 1999 a agosto de 2000. As colunas em negrito representam os meses considerados secos.

\begin{tabular}{|c|c|c|c|c|c|c|c|c|c|c|c|c|c|c|c|c|c|}
\hline \multirow{3}{*}{$\begin{array}{l}\text { Subfamílias } \\
\text { Coinobiontes }\end{array}$} & \multicolumn{8}{|c|}{1999} & \multicolumn{8}{|c|}{2000} & \multirow[t]{3}{*}{ Totais } \\
\hline & $\mathbf{M}$ & $\mathrm{J}$ & $\mathbf{J}$ & A & $S$ & $\mathrm{O}$ & $\mathbf{N}$ & $\mathrm{D}$ & $\mathrm{J}$ & $\mathrm{F}$ & $\mathrm{M}$ & A & & $\mathbf{J}$ & $\bar{J}$ & A & \\
\hline & & & & & & & & & & & & & & & & & \\
\hline Agathidinae & 1 & 1 & 0 & 2 & 3 & 5 & 1 & 6 & 0 & 2 & 4 & 2 & 0 & 1 & 2 & 3 & 33 \\
\hline Alysiinae & 1 & 0 & 3 & 2 & 1 & 0 & 0 & 3 & 4 & 2 & 4 & 1 & 5 & 0 & 1 & 5 & 32 \\
\hline Aphidiinae & 0 & 0 & 4 & 0 & 0 & 2 & 0 & 2 & 0 & 2 & 0 & 0 & 0 & 1 & 1 & 0 & 12 \\
\hline Blacinae & 0 & 0 & 0 & 0 & 0 & 0 & 0 & 1 & 0 & 1 & 0 & 0 & 0 & 0 & 0 & 0 & 2 \\
\hline Cardiochilinae & 1 & 0 & 0 & 0 & 0 & 0 & 0 & 1 & 1 & 0 & 1 & 1 & 0 & 0 & 0 & 0 & 5 \\
\hline Cenocoeliinae & 0 & 0 & 0 & 0 & 0 & 1 & 3 & 4 & 0 & 0 & 1 & 0 & 0 & 0 & 0 & 0 & 9 \\
\hline Cheloninae & 0 & 1 & 3 & 7 & 14 & 11 & 5 & 12 & 21 & 14 & 8 & 12 & 5 & 6 & 12 & 7 & 138 \\
\hline Euphorinae & 1 & 0 & 1 & 3 & 5 & 6 & 2 & 13 & 8 & 3 & 3 & 3 & 4 & 16 & 10 & 14 & 92 \\
\hline Gnamptodontinae & 0 & 0 & 0 & 0 & 1 & 0 & 0 & 1 & 0 & 2 & 0 & 0 & 3 & 1 & 1 & 2 & 11 \\
\hline Helconinae & 2 & 2 & 3 & 2 & 5 & 12 & 18 & 19 & 21 & 10 & 3 & 4 & 1 & 1 & 1 & 2 & 106 \\
\hline Homolobinae & 0 & 1 & 4 & 1 & 4 & 3 & 2 & 3 & 1 & 1 & 0 & 1 & 0 & 1 & 0 & 0 & 22 \\
\hline Ichneutinae & 0 & 0 & 0 & 0 & 1 & 0 & 0 & 0 & 1 & 0 & 1 & 0 & 1 & 0 & 0 & 1 & 5 \\
\hline Macrocentrinae & 0 & 1 & 1 & 0 & 7 & 8 & 9 & 10 & 8 & 3 & 0 & 1 & 0 & 4 & 1 & 0 & 53 \\
\hline Meteorinae & 0 & 0 & 13 & 18 & 6 & 0 & 0 & 0 & 0 & 0 & 0 & 1 & 0 & 1 & 3 & 1 & 43 \\
\hline Microgastrinae & 14 & 60 & 135 & 213 & 134 & 52 & 48 & 54 & 22 & 41 & 30 & 60 & 43 & 224 & 178 & 187 & 1495 \\
\hline Miracinae & 0 & 0 & 0 & 0 & 3 & 2 & 1 & 0 & 0 & 1 & 0 & 1 & 0 & 0 & 2 & 3 & 13 \\
\hline Opiinae & 0 & 2 & 9 & 8 & 20 & 4 & 6 & 8 & 8 & 8 & 2 & 7 & 2 & 5 & 2 & 1 & 92 \\
\hline Orgilinae & 5 & 6 & 2 & 5 & 0 & 1 & 0 & 2 & 3 & 4 & 1 & 1 & 1 & 1 & 1 & 1 & 34 \\
\hline Rogadinae & 3 & 5 & 9 & 17 & 17 & 7 & 1 & 14 & 17 & 1 & 0 & 1 & 3 & 8 & 5 & 2 & 110 \\
\hline Subtotais & 28 & 79 & 187 & 278 & 221 & 114 & 96 & 153 & 115 & 95 & 58 & 96 & 68 & 270 & 220 & 229 & 2307 \\
\hline Idiobiontes & & & & & & & & & & & & & & & & & \\
\hline Braconinae & 6 & 4 & 18 & 25 & 12 & 8 & 4 & 17 & 27 & 16 & 5 & 12 & 8 & 5 & 3 & 4 & 174 \\
\hline Doryctinae & 1 & 3 & 15 & 14 & 37 & 14 & 19 & 19 & 6 & 18 & 3 & 11 & 4 & 50 & 26 & 32 & 272 \\
\hline Hormiinae & 0 & 2 & 2 & 3 & 3 & 3 & 1 & 4 & 6 & 4 & 0 & 1 & 1 & 3 & 3 & 3 & 39 \\
\hline Subtotais & 7 & 9 & 35 & 42 & 52 & 25 & 24 & 40 & 39 & 38 & 8 & 24 & 13 & 58 & 32 & 39 & 485 \\
\hline Totais & 35 & 88 & 222 & 320 & 273 & 139 & 120 & 193 & 154 & 133 & 66 & 120 & 81 & 328 & 252 & 268 & 2792 \\
\hline
\end{tabular}


hospedeiros potenciais e os padrões de utilização dos mesmos.

\section{MATERIAL EMÉTODOS}

A área de estudo e metodologia utilizada assemelham-se às descritas em Cirelli \& Penteado-Dias (2003). Os exemplares identificados foram agrupados por táxon e sexo em cada ambiente amostrado. Foram calculados os totais mensais de cada subfamília identificada. A determinação do período seco foi feita seguindo o critério de GAUSSEN et al. apud IBGE (1977) que consideram seco aquele mês cujo total das precipitações (P), em mm, é igual ou inferior ao dobro da temperatura média (T) em graus Celsius $(\mathrm{P}=2 \mathrm{~T})$.

Análise estatística. A riqueza de subfamílias foi considerada igual ao número total de subfamília de cada amostra (ODum 1985; MagurRan 1988). Atribuiu-se N ao número de indivíduos capturados. O teste de correlação utilizado foi de Spearman. O nível de significância foi de 0,05 .

\section{RESULTADOS EDISCUSSÃO}

As cinco armadilhas capturaram 2.792 Braconidae de 22 subfamílias, das quais 19 são coinobiontes e 3 idiobiontes (Tabela I). Houve perda de material em 1999, nos meses de maio ( no cerrado e mata ciliar degradada), junho ( na mata ciliar degradada e cerrado), em agosto (mata ciliar degradada), setembro (cerrado) outubro e novembro (mata ciliar degradada); em 2000 as perdas foram em março (mata estacional semidecídua e cerrado) e maio (cerradão).
Não houve correlação entre o número de indivíduos e os dados climáticos de temperatura $\left({ }^{\circ} \mathrm{C}\right)$ (Coeficiente de Spearman $(\mathrm{rs})=0,0196, \mathrm{p}=0,941)$ e pluviosidade $\left(\mathrm{mm}^{3}\right)(\mathrm{rs}=0,0135$, $\mathrm{p}=0,959)$. JuILLET (1964) utilizando "rotary trap", mostrou que os Braconidae são mais ativos entre $20-24^{\circ} \mathrm{C}$ e em umidade em torno de $75 \%$. No período de estudo, a temperatura média foi $20,71{ }^{\circ} \mathrm{C}$ e a pluviosidade foi de $100,4 \mathrm{~mm}^{3}$, fato que deve ter influenciado no teste de correlação, pois as condições climáticas do município parecem propícias para o desenvolvimento constante das comunidades de Braconidae.

A Fig. 1 apresenta as médias mensais de temperatura $\left({ }^{\circ} \mathrm{C}\right)$ e pluviosidade $\left(\mathrm{mm}^{3}\right)$ durante o período de estudo, a distribuição e abundância dos Braconidae adultos coinobiontes e idiobiontes. Observa-se que a abundância dos indivíduos tende a ser inversamente proporcional ao aumento da temperatura e pluviosidade.

Existe uma hipótese de que nos trópicos o número de parasitóides especialistas (coinobiontes) diminui devido à fragmentação das populações de hospedeiros fitófagos, o que não acontece com os parasitóides generalistas (idiobiontes) (JANZEN 1981; AsKEW \& Shaw 1986 ). HAwKINS (1990) observou que tanto em regiões temperadas quanto nos trópicos os parasitóides especialistas estão concentrados em locais onde existem hospedeiros expostos e os generalistas em locais onde existem hospedeiros ocultos. Portanto, a distribuição de idiobiontes (generalistas) e coinobiontes (especialistas) é influenciada pelo nicho dos seus hospedeiros. As subfamílias de Braconidae mais abundantes foram, respectivamente, Microgastrinae (N=1495) (53,55\%); Doryctinae (N=272) $(9,74 \%)$;

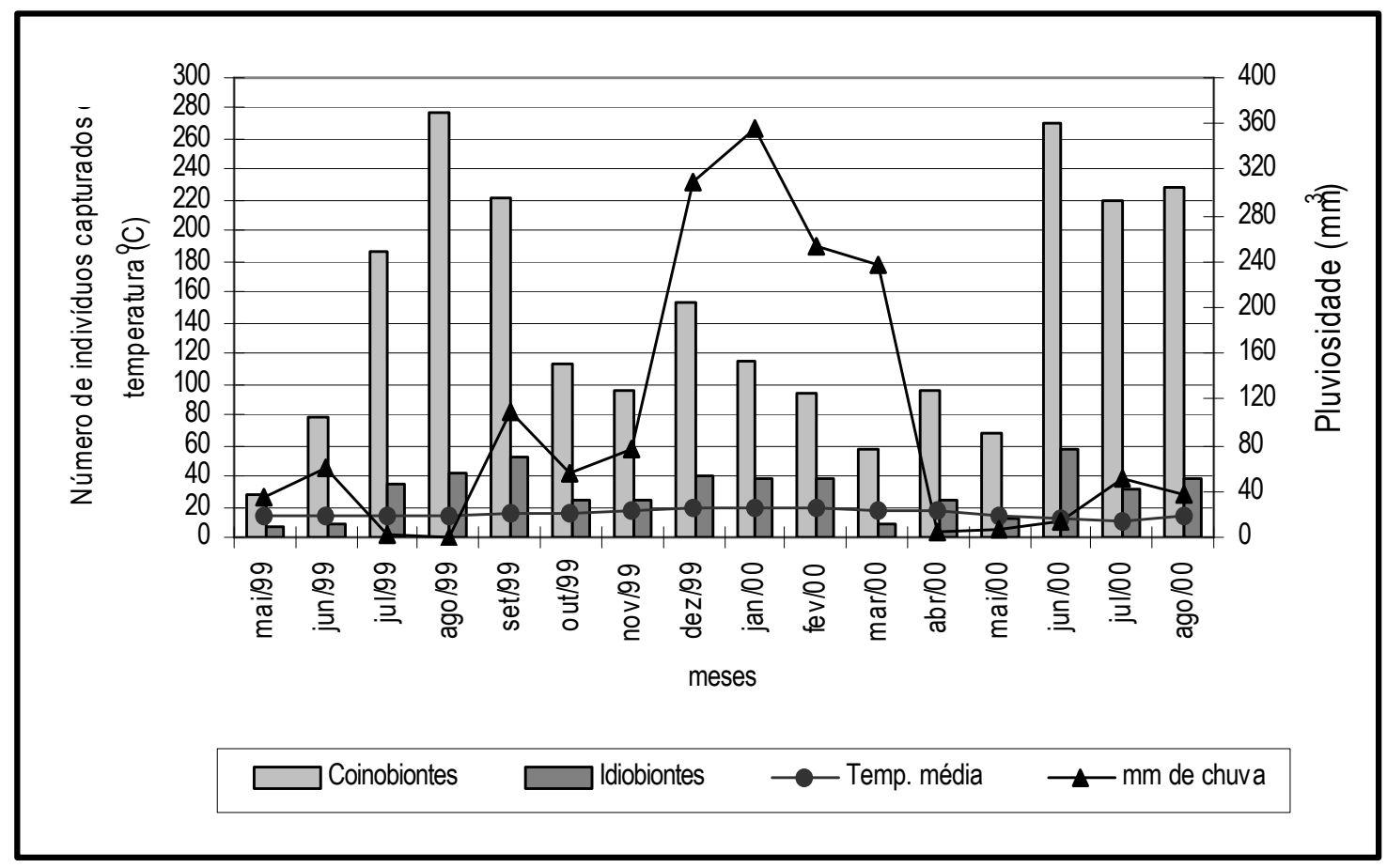

Fig. 1. Ocorrência de Braconidae adultos capturados na Área de Proteção Ambiental de Descalvado, SP por armadilha Malaise, médias de temperatura $\left({ }^{\circ} \mathrm{C}\right)$ e pluviosidade $\left(\mathrm{mm}^{3}\right)$. Período de maio de 1999 a agosto de 2000. 
Braconinae $(\mathrm{N}=174)(6,23 \%)$; Cheloninae $(\mathrm{N}=138)(4,94 \%)$; Rogadinae $(\mathrm{N}=110)(3,94 \%)$; Helconinae $(\mathrm{N}=106)(3,79 \%)$; Euphorinae ( $\mathrm{N}=92)$ e Opiinae $(\mathrm{N}=92)$ (ambos com 3,29\%) (Tabela 1). Estas comunidades representam $79,80 \%$ do todo capturado. Estudos realizados por RuIz (1989), em mata estacional semidecídua e cerradão da região de São Carlos, SP, também apontaram os Microgastrinae e Doryctinae como os mais abundantes, entretanto foram capturados por diferentes técnicas, sendo a armadilha Malaise a mais eficiente na sua coleta. Não houve coleta de hospedeiros, entretanto pode-se inferir que nos remanescentes naturais da APA de Descalvado existem insetos fitófagos externos, como lagartas herbívoras e aqueles ocultos em tecidos vegetais, como é o caso de minadores, formadores de galhas ou brocadores, pois representantes de Microgastrinae (coinobiontes) e Doryctinae (idiobiontes) foram capturadas em mais da metade de todo o universo amostrado.

Os coinobiontes mais abundantes foram Microgastrinae, Cheloninae e Rogadinae, que contribuíram com 75,55\% (N= 1743) dos indivíduos capturados (Tabela 1). Muitas espécies desses grupos são importantes componentes dos complexos de parasitóides de muitas espécies de Lepidoptera consideradas pragas de culturas agrícolas. As outras subfamílias foram agrupadas em demais coinobiontes $(\mathrm{N}=564)$ e assim analisadas. Microgastrinae é considerada uma das maiores subfamílias de Braconidae; é cosmopolita e homogênea, caracterizando-se por grande riqueza de espécies (GAULD 1991), são endoparasitóides solitários ou gregários de larvas de Lepidoptera. Usualmente, a oviposição é feita na larva jovem do hospedeiro, mas raramente o ovo pode ser atacado (WILBERT apud Ruzz 1989). Algumas espécies gregárias tecem estruturas complexas expostas contendo muitos casulos. Os Cheloninae compõem uma subfamília grande e cosmopolita; são endoparasitóides solitários de ovos e larvas de Lepidoptera e a oviposição ocorre no ovo do hospedeiro sendo o desenvolvimento da larva do parasitóide suspenso no primeiro ínstar, até que a larva hospedeira tenha tecido seu casulo pupal (Shaw \& HudDleston 1991). Os Rogadinae constituem um grupo grande e bastante heterogêneo, de distribuição mundial. Observa-se um comportamento de transição do ecto- ao endoparasitoidismo, com representantes que são idiobiontes ectoparasitóides solitários ou gregários de larvas ocultas (usualmente minadoras de folhas) de Lepidoptera, Diptera, Coleoptera e Symphyta (Hymenoptera) e representantes ectoparasitóides e endoparasitóides coinobiontes de larvas de Lepidoptera.

Os Doryctinae foram responsáveis por mais da metade $(56,08 \%)$ dos idiobiontes capturados; os Braconinae contribuíram com $35,88 \%$ e os Hormiinae com $8,04 \%$ (Tabela 1). Muitas de suas espécies são importantes no controle de pragas florestais (Doryctinae) e de agroecossistemas (Braconinae e Hormiinae). Os Doryctinae e Braconinae distribuem-se por todo o globo e provavelmente formam os grupos mais primitivos de Braconidae (Quicke \& MARSH 1992). A maioria é parasitóide solitário ou gregário de larvas ocultas (principalmente Braconinae) de Coleoptera e Lepidoptera xilófagos; os Doryctinae podem também atacar larvas ocultas de Symphyta (Hymenoptera) bem como Embioptera (SHAw \& EDGERLY 1985). Os Braconinae raramente parasitam larvas de Diptera e Symphyta e vários gêneros são endoparasitóides gregários de pupas expostas de Lepidoptera (ACHTERBERG 1984). As larvas de Doryctinae e Braconinae se alimentam sobre o hospedeiro, que é paralisado durante a oviposição; o casulo é formado nos túneis de tecidos vegetais ou outros locais ocultos (CAPEK apud Ruzz 1989). Os Hormiinae também são cosmopolitas e bastante heterogêneos. São ectoparasitóides solitários e alguns gregários de larvas ocultas de Lepidoptera, Coleoptera e menos comumente de Hymenoptera e Diptera. A larva do parasitóide se desenvolve sobre a larva hospedeira de último ínstar (AsKew \& Shaw 1986).

As Figs. 2a-c apresentam a fenologia de vôo dos Braconidae coinobiontes e idiobiontes adultos.

Na Fig. 2a, observa-se que, durante o período estudado, as duas comunidades de Braconidae apresentaram um pico em cada ano. Os coinobiontes apresentaram pico em agosto de 1999, um mês antes que os idiobiontes; em 2000 os coinobiontes e idiobiontes apresentaram pico simultâneo em junho. Nos demais meses de coleta as populações tenderam a permanecer constantes com ligeiro declínio em outubro de 1999, março e maio de 2000. Wolda (1998) observou que em média, a porcentagem de espécies de insetos dos trópicos ativos durante o ano é alta e os picos menos definidos, quando comparados aos das zonas temperadas, fato também observado neste estudo. Pode-se ainda notar que as populações de coinobiontes e idiobiontes parecem não estar em competição, provavelmente por haver riqueza de fontes energéticas e protéicas para as formas adultas, oriundas de espécimes vegetais em floração (JERVIS et al. 1993) observadas na área de estudo durante todo o período estudado; riqueza de hospedeiros (fonte de alimento para as larvas) e por explorarem nichos diferentes.

Analisando-se a fenologia de vôo da comunidade de Braconidae coinobiontes (Fig. 2b) pode-se notar que os Microgastrinae dominam. O pico dos Microgastrinae coincidiu com o do total de coinobiontes (agosto de 1999 e junho de 2000) (Fig. 2a) e o seu declínio populacional coincidiu com o discreto pico de Cheloninae e Rogadinae em janeiro de 2000 (período de muita chuva e temperaturas máximas, Fig. 1); os demais coinobiontes apresentaram pico um mês antes (dezembro de 1999). Microgastrinae, Cheloninae e Rogadinae têm Lepidoptera como hospedeiros, entretanto apresentam nichos diferentes, sendo a maioria dos Microgastrinae parasitóides primários de larvas expostas, os Cheloninae de ovos e os Rogadinae, além de utilizar outros grupos de hospedeiros, procuram larvas ocultas e apresentam hábito noturno, condições que podem estar determinando a abundância e distribuição dos mesmos.

A Fig. 2c apresenta a fenologia da comunidade idiobionte de Braconidae adultos. Dentre os idiobiontes, os Doryctinae são dominantes, sendo observado pico populacional em setembro de 1999 e junho de 2000, coincidindo com o do total de idiobiontes (Fig. 2a). Observa-se queda populacional em 


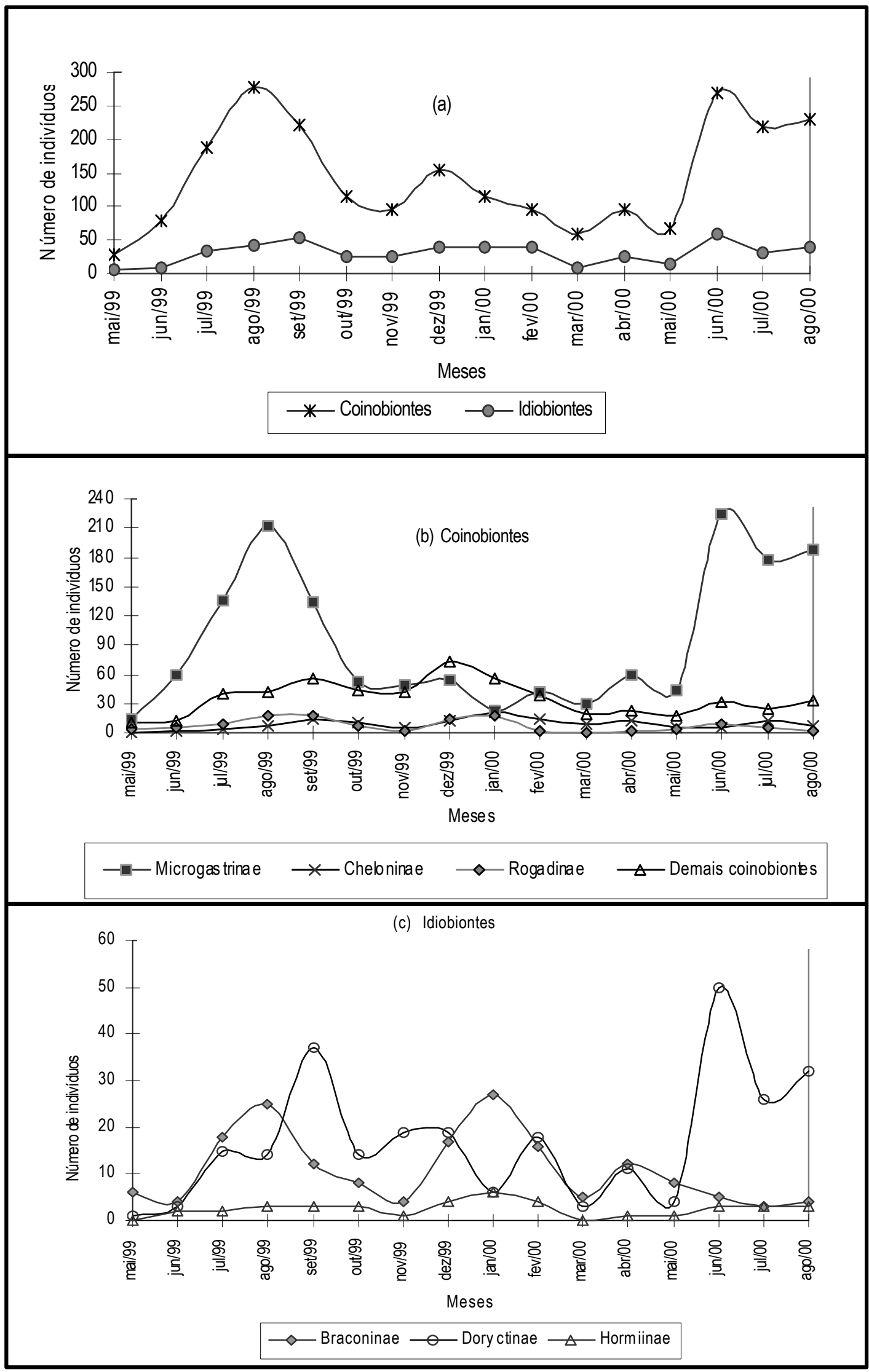

Fig. 2. Fenologia de vôo dos Braconidae capturados por armadilhas Malaise na APA de Descalvado, SP, no período de maio de 1999 a agosto de 2000. 
julho de 1999 e em janeiro de 2000, épocas dos picos de Braconinae, podendo indicar competição interespecífica entre Doryctinae e Braconinae, pois em setembro de 1999 e em junho de 2000, os Braconinae tiveram baixas taxas populacionais. Os Hormiinae mantiveram-se constantes durante o período amostrado, sendo observado ligeiro aumento populacional em janeiro de 2000, período de máximas de temperatura e pluviosidade (Fig. 1).

A proporção de fêmeas $(\mathrm{N}=1594)$ e machos $(\mathrm{N}=689)$ coinobiontes foi 2,3:1, já na comunidade idiobionte esta proporção subiu para 4,6:1 (fêmeas N=392 e machos N=85). Foi observado resíduo de 32 exemplares, por não ter sido possível fazer a sexagem. As proporções sexuais, conhecidas nos Braconidae, mostram um grande número de espécies cuja progênie tem os machos em predominância e apenas um pequeno número com a grande maioria de progênie formada por fêmeas. Por outro lado, a reprodução unissexual (partenogênese) é considerada normal em certo número de espécies de Braconidae (Clausen apud Ruiz 1989).

As Figs. 3a e b mostram a fenologia de vôo das fêmeas e machos adultos da comunidade de Braconidae coinobionte e idiobionte, respectivamente.

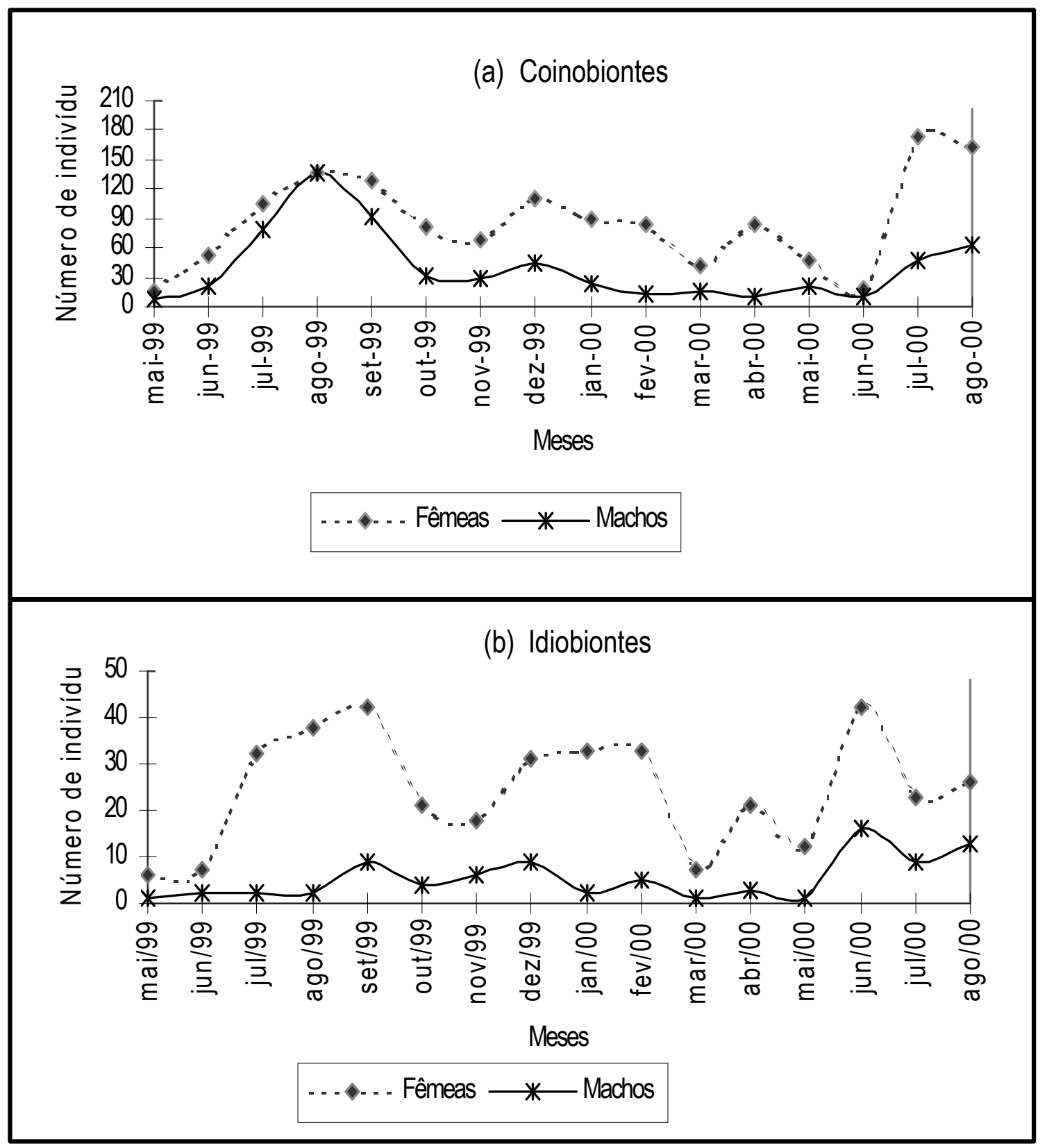

Fig. 3. Fenologia de vôo de fêmeas e machos adultos de Braconidae capturados por armadilhas Malaise na APA de Descalvado, SP, no período de maio de 1999 a agosto de 2000. 
Na Fig. 3a observam-se pico sincrônico na captura de fêmeas e machos coinobiontes entre os meses de junho e agosto de 1999. Após este período, a população de fêmeas apresentou superioridade numérica em relação à população de machos em, pelo menos, outras duas vezes. Houve queda populacional de fêmeas entre os meses de maio e junho de 2000, chegando a se equiparar com a dos machos, para logo a seguir, em julho de 2000, ambos apresentarem crescimento populacional.

Diferente dos coinobiontes, a população de fêmeas dos idiobiontes é sempre pelo menos duas vezes superior a dos machos. Seus picos e declínios coincidem com os da comunidade idiobionte (Fig. 3b).

A comunidade de Braconidae constitui fator importante na dinâmica e controle das populações de muitas espécies de insetos, principalmente Lepidoptera causadores de danos econômicos em sistemas agrícolas.

O município de Descalvado apresenta macroclima favorável ao desenvolvimento e manutenção destes Braconidae. Os remanescentes naturais, mesmo degradados, da APA de Descalvado distribuem-se pela área na forma de manchas isoladas, cercadas por uma matriz de ocupação agropecuária. Assim, a manutenção e recuperação das mesmas se torna extremamente importante para agricultura do município, do ponto de vista econômico e de qualidade ambiental silvestre e antrópica, podendo se constituir em fontes de inimigos naturais para insetos-praga que venham a se instalar nas culturas adjacentes.

Agradecimentos. Aos proprietários e funcionários das fazendas onde as armadilhas foram montadas, à CAPES, ao CNPq e à FAPESP pelo suporte financeiro.

\section{REFERÊNCIAS}

Achterberg, C. Van. 1984. Essay on the Phylogeny of Braconidae (Hymenoptera, Ichneumonoidea). Entomologisk Tidskrift 105: 41-58.

Achterberg, C. VAn. 1988. Parallelisms in the Braconidae (Hymenoptera) with special reference to the biology, p. 85-115. In: V. K. Gupta, (ed.). Advances in Parasitic Hymenoptera Research. Leiden, E. J. Brill, $546 \mathrm{p}$.

Alphen, J. J. M. Van \& L. E. M. Vet 1986. An evolutionary approach to host finding and selection, p. 23-61. In: J. Waage \& D. Greathead (eds). Insect Parasitoids. London, Academic Press, xvii+389 p.

Askew, R. R. \& M. R. Shaw. 1986. Parasitoid communities: their size, structure and development, p. 225-264. In: J. WAAGE \& D. GREATHEAD (eds.). Insect Parasitoids. London, London Academic, xxvii+389 p

Cirelli, K. R. N \& A. M. Penteado-Dias. 2003. Análise da riqueza da fauna de Braconidae (Hymenoptera: Ichneumonoidea) em remanescentes naturais da Área de Proteção Ambiental (APA) de Descalvado, SP. Revista Brasileira de Entomologia 47(1): 89-98
Dover, B. A. \& S. B. Vinson. 1990. Effect of host logarion and starvation on the development and emergence of the parastoid Campoletis sonorensis. Entomologia Experimentalis et Aplicata 57(3): 209213.

GAuLD, I. D. 1987. Some factors affecting the composition of tropical ichneumonid faunas. Biological Journal of the Linnean Society 30: $299-312$.

Gauld, I. D. \& B. Bolton. 1988. The Hymenoptera. Oxford, Oxford University Press, $332 \mathrm{p}$.

Gauld, I. D. 1991. The Ichneumonidae of Costa Rica, 1. Memoirs of the American Entomological Institute 47: 1-589.

Godfray, H. C. J. 1994. Parasitoids: behavioral and evolutionary ecology. New Jersey, Princeton University Press, 437 p.

Hawkins, B. A. 1990. Global patterns of parasitoid assemblage size. Journal of Animal Ecology 59: 57-72.

IBGE. 1977. Fundação Instituto Brasileiro de Geografia e Estatística. Geografia do Brasil: Região Centro-Oeste. Rio de Janeiro, FSERGRAF 4: $364 \mathrm{p}$.

JANZEN, D. H. 1981. The peak in North American Ichnemonid speciesrichness lies between $38^{\circ}$ and $42^{\circ} \mathrm{N}$. Ecology 62: 532-537.

Jervis, M. A.; N. A. Kidd; M. G. Fitton; T. Huddleston \& H. A. Dawah. 1993. Flower-visiting by hymenopteran parasitoids. Journal of Natural History 27: 67-105.

Juillet, J. A. 1960. Some factors influencing the flight activity of hymenopterous parasites. Canadian Journal of Zoology 38: 10571061.

JuILLET, J. A. 1964. Influence of weather on flight activity of parasitic hymenoptera. Canadian Journal of Zoology 42: 1133-1141.

Lewis, C. N. \& J. B. Whitfield. 1999. Braconid wasp (Hymenoptera: Braconidae) diversity in forest plots under different silvicultural methods. Environmental Entomology 28(6): 986-997.

MagurRan, A. E. 1988. Ecological diversity and its measurement. Princeton, Princeton University Press, 179 p.

Nealis, V. G. 1988. Weather and ecology of Apanteles fumiferanae Viereck (Hymenoptera: Braconidae). Memoirs of the Entomological Society of Canada 146: 57-70.

Odum, E. P. 1985. Ecologia. Rio de Janeiro, Ed. Interamericana, 434 p.

Quicke, D. L. \& P. M. Marsh. 1992. Two new species of Neotropical parasitic wasps with highly modified ovipositor (Hymenoptera, Braconidae: Braconinae and Doryctinae). Proceedings of the Entomological Society of Washington 94: 559-567.

Ruiz, S. S. 1989. Aspectos biológicos e abundância sazonal de Microgastrinae (Hymenoptera, Braconidae) na região de São Carlos - SP. Dissertação de Mestrado. Universidade Federal de São Carlos, $124 \mathrm{p}$.

SALt, G 1968. The resistence of insect parasitoids to the defence reactions of their hosts. Biological Reviews of the Cambridge Philosophical Society 43: 200-232.

Shaw, M. R. \& J. S. EdGerLy. 1985. A new braconid genus (Hymenoptera) parasitizing web-spinner (Embiidina) in Trinidad. Psyche 92: 505511.

Shaw, M. R. \& T. Huddeston. 1991. Classification and biology of Braconidae wasps (Hymenoptera: Braconidae). Handbooks for the Identification os British Insects. Part 11. Royal Entomological Society of London 7: 1-126.

Thangavelu, K. 1993. Natural enemies of muga silkworm, Antheraea assama Wetswood (Lepidoptera: Saturniidae). Journal of Insect Science 6: $57-59$.

Vinson, S. B. 1981. Habitat location, p. 51-57. In: J. WAAGed \& D. GREATHEAD (eds.). Insect parasitoids. London, London Academic, xxvii+389 p.

Wolda, H. 1988. Insect seasonality: why? Annual Review of Ecology and Systematics 19: 1-18. 